\title{
Guest editorial for spatial agent-based models: current practices and future trends
}

\author{
Alison Heppenstall ${ }^{1,2} \cdot{\text { Andrew } \text { Crooks }^{3,4}}^{3}$ \\ Published online: 28 March 2019 \\ C Springer Science+Business Media, LLC, part of Springer Nature 2019
}

Over the last few years we have seen spatial agent-based modelling beginning to bridge the gap from cautious early adoption towards general acceptance within the geographical sciences. One of the key features that has contributed to this is its ability to represent individual characteristics and behaviours. Through building simulations that exploit these characteristics, researchers have been given a new tool to simulate and understand the consequences of individual interactions, behaviours and movements through space and time. This has allowed questions that are of interest to both academics and policy makers to be posed, for example: what would be the economic impact of water reform? How might pedestrians react in a disaster? Who uses our urban spaces at different times of day? What would be the impact if funding was changed for academic research? What will be the impact of changing how prices NASDAQ where recorded? How might fish populations be impacted in changing water conditions? Or how might a certain disease spread? (for more details on such questions see [1-5]).

This rise in popularity has been aided by technological advances such as the emergence of accessible development platforms, greater processing power and storage capacity [6]. However, it could also be argued that the proliferation and availability of new and novel forms of micro-level data have equally contributed to its uptake [7]. Social scientists find themselves, for the first time, in a data rich era with access to a variety of detailed geo-referenced data sources (e.g., social media, mobile phone data, census information, etc.) that have the potential

Alison Heppenstall

a.j.heppenstall@leeds.ac.uk

Andrew Crooks

acrooks2@gmu.edu

1 School of Geography, University of Leeds, Leeds LS2 9JT, UK

2 Alan Turing Institute, London NW1 2DB, UK

3 Department of Computational and Data Sciences, George Mason University, Fairfax, VA 22030, USA

4 Department of Geography and Geoinformation Science, George Mason University, Fairfax, VA 22030, USA 
to reveal how and when, individuals are using spaces. These data allow more complex questions to be posed and answered, but more generally, allow researchers to gain new and deeper insights into complex spatial systems. Part of the focus of this special issue is to ask questions about how agent-based modelling approaches have lent themselves to simulating the dynamics of spatial processes. What has been, or will be, the influence of "big data" on our ability to understand and simulate spatial systems? What is the appropriate level of spatial analysis and temporal scale to capture and model spatial phenomena?

While the development of agent-based modelling can be described as haphazard, with advances occurring within many disciplinary silos, there have been some notable methodological successes. Grimm et al.'s Overview Design concepts Details (ODD) protocol [8] - and recent additions such as ODD+D (Decision) [9] and ODD+2D (Decisions and Data) [10] - has standardised how agent-based models are presented within research, having a positive impact on the replicability of models. There has also been a notable rise in code sharing and open source repositories, for example Github and sites such as https://www.comses.net/ (formally openabm.org). However, the sharing of models and data is still not the norm amongst most researchers. While progress has been made in some areas of agent-based modelling, there has been a lack of progress in the area of model development, these range from model initialisation to that of derivation of rule sets from different forms of data, creating large-scale simulations, to that of calibration and robust validation [11]. Validation of agent-based models remains a challenging area, as Polhill et al. [12] neatly articulate in their paper in this issue: "There is a clear, qualitative difference between adjusting parameters of a mathematical function until it fits some data, and replicating those data as emergent phenomena of the interactions among heterogeneous agents".

The call that was put out for this special issue asked for papers that not only looked at current trends but also highlighted and addressed the advances and challenges that researchers working within the area of spatial agent-based models face. Several common themes became apparent when reviewing the articles. All the authors were in agreement that while there has been a noticeable uptake in agent-based modelling, more work is needed to bridge the gap to acceptance as a standard tool within the spatial sciences. Data (variable quality and availability) was an issue that was discussed by almost all of the authors, particularly how to translate high quality data into models to create behavioural rules and the use of novel forms of data to calibrate an empirical model (e.g. [13]). How to represent and simulate behaviour in agentbased models was also a recurrent issue with two papers discussing how approaches borrowed from machine learning can be used to improve the representation of behaviour [14, 15]. How to create models that could scale from the micro to macro was another theme with the point being made that current agent-based modelling architectures do not foster models that are easily translatable to a regional or global context (e.g. [17]), nor are interactions across scales adequately addressed in most models (e.g. [16]).

The Polhill et al. [12] paper within this special issue covers with alacrity the main issues and challenges that agent-based modelling needs to address to bridge the gap to total acceptance. The authors present a "tube map" for agent-based models that emphasises the connections needed with other endeavours, including the type of cross-disciplinary work that is required. Polhill et al. [12] identify and discuss several areas where they feel advances are needed in order for agent-based modelling to become more established. These include validation, ontologies to describe the systems and data needs in empirical and applied contexts. Using new forms of data to calibrate and simulate movements of a daytime (ambient) population is an issue that Crols and Malleson [13] grapple with using an empirical agent-based model. Their 
goal here is to use new forms of data to create insights into how ambient populations move around and use urban spaces. The data in question are daily footfall collected from a Wi-Fi sensor network, a form of dynamic individual-level data that will only become increasingly prevalent in the future.

The issue of how to represent behaviour and decision-making in agent-based modelling received the most attention in the papers that were submitted. This is hardly surprising as simulating behaviour is at the heart of agent-based modelling. Runck et al. [14] argue, echoing the views of many within the agent-based modelling community, that agent-based models need high quality behavioural data and a transparent means of translating these data into models that allows for reproducibility. The solution that they suggest is natural language processing and Bayesian learning that would facilitate the creation of agents who can construct mental models via interactions with other agents and the environment. This approach would provide a new way to model agent cognitive processes for a broad set of human-environmental processes. Methods from machine learning are beginning to gain traction within agent-based modelling community, but these approaches are computationally intensive and often require large amounts of data. The potential benefits of machine learning for helping to simulate behavioural change is recognised by Abdulkareem et al. [15]. They identify that one of the issues with accurately simulating behaviour is the large number of micro-level data sets that are required. Within their paper, they neatly demonstrate how Bayesian networks can be used with limited data sets to get around this problem.

Lippe et al. [16], in their 'thought piece' addresses the issue of scale within agent-based models. They make the argument that when considering a spatially-explicit domain, current agent-based modelling architectures are not easily translatable to a regional or global context, nor are interactions across scales adequately addressed. They highlight the need for agentbased modelling to cross the gap between micro-scale actors and larger-scale environmental and political structures in a way that can incorporate sufficient realism in a spatial and temporal analysis that can be useful for policy analysis. Looking at the issue of scale from a different perspective, Taillander et al. [17] offer a way to translate from a local to a regional context. They observe that while the rise of software platforms allows non-computer scientists to create increasingly complex and detailed small to mid-size simulations, very few are adapted to largescale simulations. To this end, they present the capabilities associated with an extension of GAMA - a platform that readily allows the development of complex large-scale spatial models.

Within this special issue, the papers present not only a great synthesis of the current practices but also several of the key advances and challenges within the realm of spatial agent-based modelling are brought to bare. However, it is clear that to cross the bridge from novel tool to full acceptance as a standard tool within the geographical sciences, spatial agent-based modelling still has some way to go. Nevertheless, there are a number of positive avenues of research emerging. The benefits of machine learning approaches (as alluded to earlier) integrated into agent-based models are beginning to be felt within geography with a number of developments such as dynamic data assimilation [18], spatial learning [19] and reinforcement learning [20, 21] starting to appear in the literature. Approaches not commonly associated with spatial agent-based models also have the potential to advance the field. For example, directed acyclic graphs, which are routinely used in statistical epidemiology to quantify the relationship between variables have the potential to inform agent-based models [22], especially when the number of 
variables in models and/or data grow rapidly. Today agent-based modelling is beginning to occupy a position where it can have an impact on policy and as Squazzoni [23] notes, this is important if the field is to advance. The papers in this special issue can therefore be seen as a stepping stone towards this.

\section{References}

1. Government Office for Science (2018) Computational Modelling: Technological Futures. Government Office for Science, London

2. Darley V, Outkin AV (2007) NASDAQ Market Simulation: Insights on a Major Market from the Science of Complex Adaptive Systems. World Scientific Publishing, River Edge

3. Railsback SF, Harvey BC (2002) Analysis of habitat selection rules using an individual-based model. Ecology 83(7):1817-1830

4. Waldrop MM (2018) Free Agents. Science 360(6385):144-147

5. Moussaïd M, Helbing D, Theraulaz G (2011) How simple rules determine pedestrian behavior and crowd disasters. Proc Natl Acad Sci 108(17):6884-6888

6. Malleson N, Heppenstall A, Crooks AT (2018) Place-based simulation modelling: agent-based modelling and virtual environments. In: Oxford Research Encyclopedia of Criminology and Criminal Justice. Oxford University Press, Oxford University, UK. https://doi.org/10.1093/acrefore/9780190264079.9780 190264013.9780190264319

7. Crooks AT, Malleson N, Wise S, Heppenstall A (2018) Big data, agents and the City. In: Schintler LA, Chen Z (eds) Big data for urban and regional science. Routledge, New York, pp 204-213

8. Grimm V, Berger U, Bastiansen F, Eliassen S, Ginot V, Giske J, Goss-Custard J, Grand T, Heinz S, Huse G, Huth A, Jepsen J, Jorgensen C, Mooij W, Muller B, Pe'er G, Piou C, Railsback S, Robbins A, Robbins M, Rossmanith E, Ruger N, Strand E, Souissi S, Stillman R, Vabo R, Visser U, Deangelis D (2006) A standard protocol for describing individual-based and agent-based models. Ecol Model 198(1-2):115-126

9. Müller B, Bohn F, Dreßler G, Groeneveld J, Klassert C, Martin R, Schlüter M, Schulze J, Weise H, Schwarz N (2013) Describing human decisions in agent-based models - ODD + D, an extension of the ODD protocol. Environ Model Softw 48:37-48

10. Laatabi A, Marilleau N, Nguyen-Huu T, Hbid H, Babram MA (2018) ODD+ 2D: an ODD based protocol for mapping data to empirical ABMs. J Artif Soc Soc Simul 21(2):9

11. Crooks AT, Malleson N, Manley E, Heppenstall AJ (2019) Agent-based modelling and geographical information systems: a practical primer. Sage, London

12. Polhill JG, Ge J, Hare MP, Matthews KB, Gimona A, Salt D, Yeluripati J (2019) Crossing the chasm: a 'tube-map'for agent-based social simulation of policy scenarios in spatially-distributed Systems. GeoInformatica. https://doi.org/10.1007/s10707-10018-00340-z

13. Crols T, Malleson N (2019) Quantifying the ambient population using hourly population footfall data and an agent-based model of daily mobility. Geoinformatica

14. Runck BC, Manson S, Shook E, Gini M, Jordan NR (2019) Using word Embeddings to generate datadriven human agent decision-making from natural language. Geoinformatica

15. Abdulkareem SH, Mustafa YT, Augustijn E-W, Filatova T (2019) Bayesian networks for spatial learning: a workflow on using limited survey data for intelligent learning in spatial agent-based models. Geoinformatica

16. Lippe M, Bithell M, Gotts N, Natalini D, Barbrook-Johnson P, Giupponi C, Hallier M, Hofstede GJ, Le Page C, Matthews RB, Schlüter M (2019) Using agent-based modelling to simulate social-ecological systems across scales. GeoInformatica. https://doi.org/10.1007/s10707-10018-00337-10708

17. Taillandier P, Gaudou B, Grignard A, Huynh QN, Marilleau N, Caillou P, Philippon D, Drogoul A (2019) Building, composing and experimenting complex spatial models with the GAMA platform. GeoInformatica. https://doi.org/10.1007/s10707-10018-00339-10706

18. Ward JA, Evans AJ, Malleson N (2016) Dynamic calibration of agent-based models using data assimilation. Open Science 3(4):150703

19. Manley E (2016) Estimating the topological structure of driver spatial knowledge. Appl Spat Anal Policy 9(2):165-189

20. Banino A, Barry C, Uria B, Blundell C, Lillicrap T, Mirowski P, Pritzel A, Chadwick MJ, Degris T, Modayil J, Wayne G (2018) Vector-based navigation using grid-like representations in artificial agents. Nature 557(7705):429-433 
21. Bone C, Dragicevic S (2010) Simulation and validation of a reinforcement learning agent-based model for multi-stakeholder Forest management. Comput Environ Urban Syst 34(2):162-174

22. Arnold KF, Harrison WJ, Heppenstall AJ, Gilthorpe MS (2018) DAG-informed regression modelling, agent-based modelling and microsimulation modelling: a critical comparison of methods for causal inference. Int J Epidemiol 48(1):243-253

23. Squazzoni F (2010) The impact of agent-based models in the social sciences after 15 years of incursions. Hist Econ Ideas 18(2):197-233

Publisher's note Springer Nature remains neutral with regard to jurisdictional claims in published maps and institutional affiliations. 